\title{
Belgeo
}

Revue belge de géographie

$1 \mid 2002$

Miscellaneous : Africa

\section{Dimensions régionales de la pauvreté au Sénégal}

The regional dimensions of poverty in Senegal

\section{Amadou M. Camara}

\section{(2) OpenEdition}

\section{Journals}

Édition électronique

URL : https://journals.openedition.org/belgeo/15432

DOI : 10.4000/belgeo.15432

ISSN : 2294-9135

\section{Éditeur :}

National Committee of Geography of Belgium, Société Royale Belge de Géographie

\section{Édition imprimée}

Date de publication : 30 mars 2002

Pagination : 17-28

ISSN : $1377-2368$

\section{Référence électronique}

Amadou M. Camara, « Dimensions régionales de la pauvreté au Sénégal », Belgeo [En ligne], 1 | 2002, mis en ligne le 31 mars 2002, consulté le 14 juin 2021. URL : http://journals.openedition.org/belgeo/ 15432 ; DOl : https://doi.org/10.4000/belgeo.15432

Ce document a été généré automatiquement le 14 juin 2021.

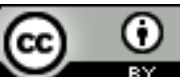

Belgeo est mis à disposition selon les termes de la licence Creative Commons Attribution 4.0 International. 


\title{
Dimensions régionales de la pauvreté au Sénégal
}

The regional dimensions of poverty in Senegal

\author{
Amadou M. Camara
}

1 La pauvreté fait présentement l'objet de plusieurs études de par le monde. Au Sénégal, quelques publications lui ont été consacrées au cours de ces dernières années. Elles l'appréhendent avec des indicateurs variables, sous des maillages différents. Des recoupements entre les mesures de la pauvreté sont nécessaires pour approcher la réalité, dont la perception à des échelles variées permettrait un traitement adapté.

\section{Le cadre théorique}

2 «La pauvreté ? Vous l'avez rencontrée devant ma porte. Regardez la maison, comptez les trous. Regardez mes affaires et les vêtements que je porte. Regardez tout ce qu'il y a ici et écrivez ce que vous voyez. C'est ça, la pauvreté !» Ainsi répondait « un homme pauvre » du Kenya aux enquêteurs de l'étude La parole aux pauvres (Banque Mondiale, 2001). Il s'agit là de la perception qu'un pauvre a de sa situation. La méthode de saisie de cette perception procède de l'approche dite «subjective » de la pauvreté. C'est une méthode participative. Elle fait désormais partie de la panoplie des méthodes utilisées pour étudier la pauvreté, depuis qu'en 1999 la Banque Mondiale a validé les propositions faites en ce sens par certains chercheurs (Robb, 1992; Pradhan et Ravallion, 1998 ; Ravallion et Lokshin, 1999).

3 L'approche «subjective» de la pauvreté vient ainsi compléter et enrichir les «démarches objectives» (Clark et al., 1981; Lachaux, 1999), qui manient divers indicateurs, monétaires ou non. Elle peut parfois se substituer à celles-ci, selon les écoles et les objectifs de recherche.

4 Au Sénégal, la plupart des études sur la pauvreté privilégient l'outil statistique et utilisent des indicateurs simples, comme le revenu per capita, le taux de scolarisation, le taux d'alphabétisation des adultes, l'accès aux services de santé, l'accès à l'eau 
potable, etc. (Enquête sur les priorités - ESP -, Direction de la Prévision et de la Statistique, $1991^{1}$; Enquête sénégalaise auprès des ménages - ESAM -, Direction de la Prévision et de la Statistique, $1994^{2}$; Enquêtes démographiques et santé - EDS - I, II, III, Direction de la Prévision et de la Statistique, 1992, 1996 et 1998. $\left.{ }^{3}\right)$. Elles s'inscrivent dans l'approche objective, positiviste. Ces études sur la pauvreté sont marquées, comme souvent, par un déficit de perception spatiale : l'examen de la différenciation spatiale de la pauvreté s'appuie sur un maillage trop large pour filtrer efficacement l'information. L'ESP et l'ESAM ne permettent d'appréhender l'opposition villecampagne qu'à l'échelle du pays.

5 La présente étude se propose d'étudier les variations régionales de la pauvreté au Sénégal sur base des résultats d'une étude réalisée par le Programme des Nations-Unies pour le Développement (1998), qui examine les variations régionales de l'indice de développement humain (IDH) ${ }^{4}$ entre les dix régions administratives du pays, maillage qui reste néanmoins grossier, d'en analyser les déterminants et d'identifier des stratégies susceptibles de résorber ces variations.

6 Une question essentielle qui se pose est de savoir si la pauvreté est plus rurale qu'urbaine, et dès lors si les variations régionales reproduisent celles du taux d'urbanisation. Les régions où l'IDH est le plus faible sont-elles aussi celles dont on émigre le plus vers celles considérées comme plus riches? Comment s'articulent régionalement les variations du développement économique et celles d'indicateurs comme le revenu des ménages, l'alphabétisation des adultes, le taux de mortalité infanto-juvénile et le niveau d'instruction des femmes ?5 L'examen de variables comme le taux de ruralité et le taux de migration nette enrichit-il les informations que l'IDH fournit quant aux différenciations régionales et comment peut-on les intégrer pour appréhender finement le phénomène de pauvreté au Sénégal?

\section{La pauvreté, phénomène plus rural qu'urbain ?}

\section{Les études nationales mettent en évidence un phénomène de pauvreté plus rural qu'urbain}

7 L'indicateur monétaire, le revenu per capita moyen est 3,6 fois plus élevé dans les villes que dans le monde rural. Ce dernier est même inférieur à la moitié du revenu national moyen par habitant. Une même situation de pauvreté plus rurale qu'urbaine est reconnue par la Banque Mondiale dans beaucoup de pays en développement (Banque Mondiale, 2001). On constate qu'avec 0,74 US\$ par jour et par personne, le revenu moyen des populations rurales sénégalaises - soit $60 \%$ de la population nationale - se situe en deçà du seuil de la pauvreté de 1 US\$ par jour proposé par la Banque Mondiale, 2001. Ils partagent cette situation avec $20 \%$ de la population de la planète et $25 \%$ de la population totale de l'Afrique subsaharienne (Banque Mondiale, 2001). En outre, le revenu moyen annuel du Sénégalais (561,9 US\$, soit 1,54 US\$ par jour) est évidemment loin de refléter la situation du plus grand nombre !

8 Les indicateurs non monétaires du tableau 1 confirment ces écarts entre le milieu urbain et les zones rurales. Il en est ainsi du taux de mortalité infanto-juvénile pour lequel la moyenne rurale sénégalaise $(17,1 \%)$ n'est pas très éloignée de celle observée dans les pays les plus pauvres du monde (environ $20 \%$ ) (Banque Mondiale, 2001), alors que la situation apparaît meilleure si l'on ne considère que la moyenne nationale de 
$14,5 \%$. Les taux respectifs d'alphabétisation rurale et d'instruction des femmes corroborent ces constats. Ils confirment aussi la relation de causalité que de nombreuses études ont nouée entre le taux général d'alphabétisation, le niveau d'instruction des femmes et le taux de mortalité infanto-juvénile (Direction de la Prévision et de la Statistique, 1992, 1996, 1998).

Tableau 1. Opposition ville-campagne à travers quelques variables issues d'enquêtes.

$\begin{array}{lccc} & \text { Ville } & \text { Campagne } & \text { Sénégal } \\ \text { Revenu par habitant (a) } & \begin{array}{c}506 \text { 483 FCFA } \\ (990,9 \text { USS) }\end{array} & \begin{array}{c}138137 \text { FCFA } \\ (270,26 \text { USS })\end{array} & \begin{array}{c}287200 \text { FCFA } \\ (561,9 \text { USS })\end{array} \\ \begin{array}{l}\text { Taux d'alphabétisation (b) } \\ \begin{array}{l}\text { Niveau d'instruction } \\ \text { des femmes (c) }\end{array}\end{array} & 52 \% & 17,5 \% & 32,9 \% \\ \begin{array}{l}\text { Taux de mortalité infanto- } \\ \text { juvénile (d) }\end{array} & 25,7 \% & 2,4 \% & 12,7 \% \\ & 9,21 \% & 17,12 \% & 14,50 \%\end{array}$

Sources :

(a) Données de l'ESP 1991, actualisées et corrigées par l'auteur à partir des estimations de la DPS sur le revenu moyen per capita au cours de la période 1993 - 1999. Le cours moyen du US\$ est estimé à 511,126 au cours de cette période.

(b) ESAM 1995, taux moyen d'alphabétisation des personnes âgées de 14 ans et plus.

(c) EDS III, 1998, pourcentage des femmes ayant un niveau d'instruction supérieur ou égal à la fin du cycle secondaire.

(d) EDS III, 1998.

9 Reprenant cette fois les valeurs régionales de l'IDH calculées dans l'étude du PNUD, le tableau 2 montre que, même à Dakar, cet indice reste inférieur à la moyenne des pays en développement. En dehors de la capitale, seule la région de Ziguinchor dépasse l'indice national moyen. Parmi les autres régions, les disparités régionales sont très fortes et on peut y distinguer trois catégories de régions (fig. 1) :

-celles proches de la moyenne nationale, dans l'ouest et le nord: Kaolack, Saint-Louis, Thiès et Fatick ;

- des régions centrales intermédiaires, Louga et Diourbel;

- les régions les plus pauvres, dans le sud-est, Tambacounda et Kolda.

- Tableau 2. Répartition régionale de l'IDH, part de la population rurale et bilans migratoires.

$\begin{array}{lccc}\text { IDH } & \begin{array}{c}\text { Pourcentage de } \\ \text { population rurale }\end{array} & \begin{array}{c}\text { Taux de migration } \\ \text { nette (1988-1992) }\end{array} & 0,52 \% \\ \text { Dakar } & 0,438 & 3,4 \% & 0,85 \% \\ \text { Diourbel } & 0,262 & 78,2 \% & -1,42 \% \\ \text { Fatick } & 0,301 & 88,1 \% & -0,34 \% \\ \text { Kaolack } & 0,315 & 75,4 \% & -0,46 \% \\ \text { Kolda } & 0,213 & 88,2 \% & -1,85 \% \\ \text { Louga } & 0,277 & 82,1 \% & -0,80 \% \\ \text { Saint-Louis } & 0,307 & 70,4 \% & 0,29 \% \\ \text { Tambacounda } & 0,222 & 83,0 \% & -0,98 \% \\ \text { Thiès } & 0,301 & 62,7 \% & -1,25 \% \\ \text { Ziguinchor } & 0,385 & 56,9 \% & - \\ \text { SENEGAL (moyenne) } & 0,342 & 58,3 \% & \end{array}$

Sources : Programme des Nations unies pour le Développement, 1998 ; CERPOD, 1997 ; Direction de la Prévision et de la Statistique, 1998. 
Figure 1. Les régions sénégalaises, leur IDH, leur taux de ruralité et leur niveau global de pauvreté.

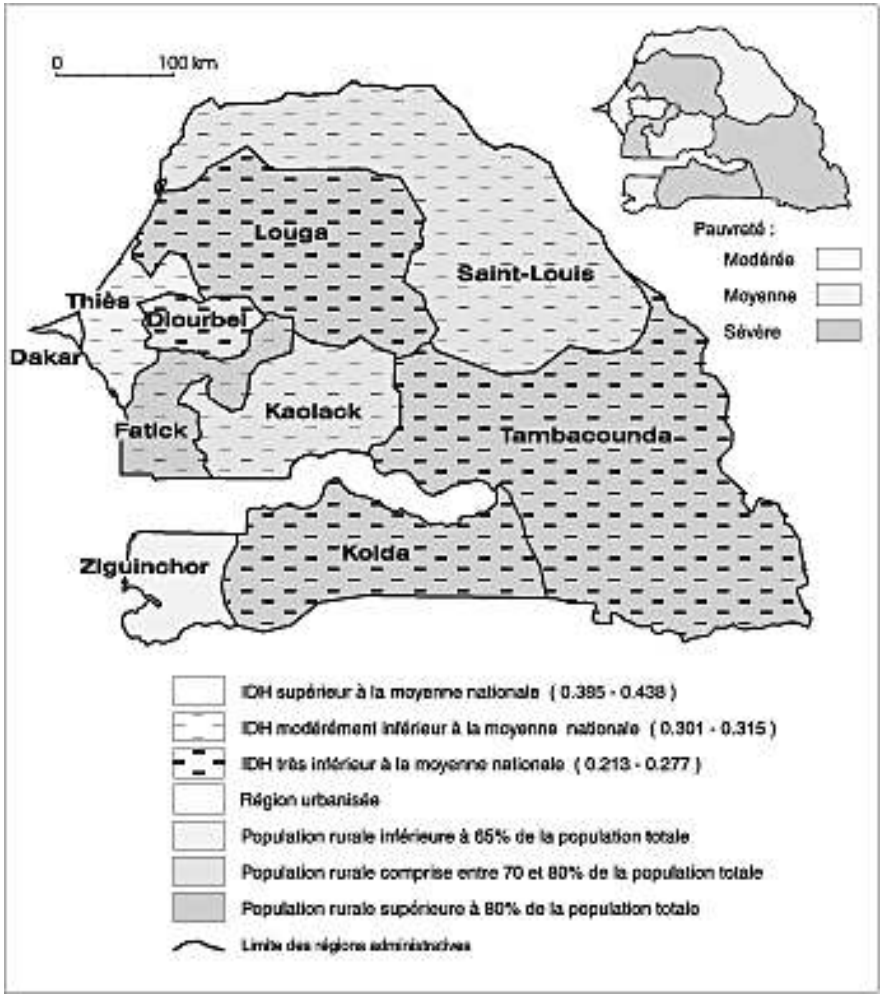

10 Il serait tentant d'invoquer le climat pour expliquer la moins bonne position des régions administratives de Louga et de Diourbel, qui coïncident peu ou prou avec les limites du désert du Ferlo. Cependant la situation du sud-est, à plus «faible développement humain » que ces deux régions, et au climat des plus humides du pays, incite à écarter tout déterminisme de cette nature. Par contre, on peut comparer la carte des variations régionales de l'IDH à celle du réseau urbain du Sénégal (fig.2). Celui-ci apparaît très dense dans les régions où l'IDH est plus élevé (Dakar, Ziguinchor), moins serré dans les régions du centre-ouest ayant un IDH intermédiaire et presque inexistant à l'est et au sud-est à plus "faible développement humain». Touba, cité religieuse dont l'urbanisation est très rapide, relativise la situation des régions de Diourbel et Louga. 
Figure 2. Le réseau urbain du Sénégal.

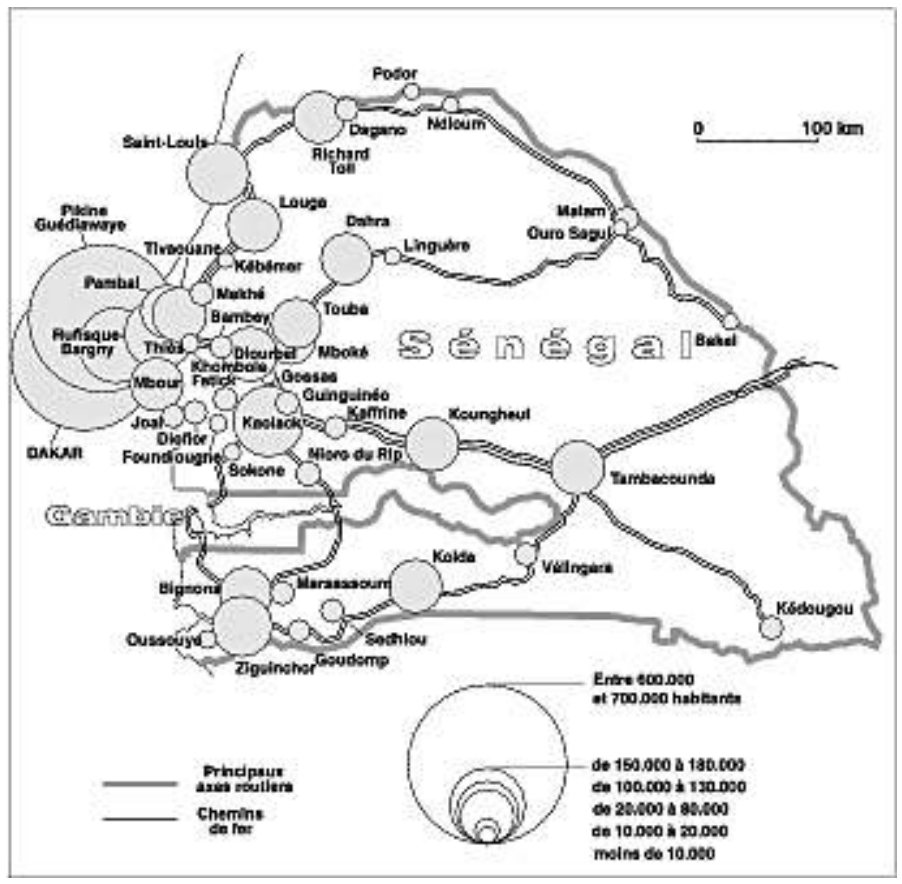

(d'après Ba, 1995)

11 Le « développement humain » d'une région aurait donc bien tendance à être d'autant plus faible que celle-ci est plus rurale. On peut étayer cette proposition en comparant les indicateurs régionaux de développement humain avec les pourcentages de population rurale de chaque région (tableau 2). Cette comparaison laisse apparaître trois cas de figure :

- les régions dont l'IDH est supérieur à la moyenne nationale et la part de ruraux inférieure à $60 \%$; ce sont celles de Dakar et de Ziguinchor ;

- les régions dont l'IDH avoisine 0,300, avec un pourcentage de ruraux compris entre 60 et $75 \%$ : Kaolack, Saint-Louis et Thiès ;

- les régions dont l'IDH est inférieur à 0,300 et le pourcentage de ruraux supérieur à $75 \%$; il s'agit des régions de Louga, Diourbel, Tambacounda et Kolda.

Avec un IDH de 0,301 et 88,1\% de ruraux, la région de Fatick n'entre dans aucune des catégories ci-dessus. Le fait est assez singulier pour que l'on y revienne.

On observe donc une corrélation inversement proportionnelle entre les variations régionales de l'IDH et le caractère rural des régions (fig. 1). Le coefficient de corrélation par rang de Spearman ${ }^{6}$ entre les deux variables est significatif, avec une valeur de $-0,644$. L'analyse régionale confirme donc bien que le fléau de la pauvreté apparaît comme un phénomène plus rural qu'urbain au Sénégal.

\section{Pauvreté rurale et mouvements migratoires}

Cette pauvreté plus grande du monde rural influence-t-elle les mouvements migratoires?

Dans une étude parue en 1997, le Centre d'Etudes et de Recherche sur la Population et le Développement (CERPOD) a calculé le bilan migratoire des régions administratives du 
Sénégal pour la période 1988-1992 (tableau 2). Il en ressort que toutes les régions du Sénégal ont un taux de migration nette négatif, à l'exception de celles de Dakar ${ }^{7}$, Diourbel et Tambacounda. La capitale du Sénégal et sa région sont donc les principales bénéficiaires de l'exode rural, les situations des régions de Diourbel et de Tambacounda étant plus atypiques.

La situation de Diourbel pourrait être liée à l'explosion démographique de la ville sainte de Touba. Plusieurs estimations concordantes (Direction de la Prévision et de la Statistique, 1992, 1996, 1998 ; Centre d'Etudes et de Recherche sur la Population et le Développement, 1997) font de la capitale du mouridisme la deuxième «ville» du Sénégal par sa population. La cité religieuse n'est plus seulement un lieu de pèlerinage ; le développement de l'économie informelle y attire un nombre de plus en plus élevé de ruraux en provenance notamment des régions voisines.

Région à la fois très rurale et à très faible niveau de développement humain, Tambacounda apparaît paradoxalement comme un pôle d'attraction. Sous l'impulsion notamment de guides religieux de la confrérie mouride, les « marabouts de l'arachide ", un front pionnier s'est ouvert dans cette région pour la conquête d'un nouvel espace agricole appelé « terres neuves ». Le développement de la culture du coton et la mise en valeur des bassins du Sénégal et de la Falémé y offrent aussi de nouvelles sources de revenus, tant aux paysans des régions voisines qu'à ceux des pays frontaliers (Mauritanie, Mali, Gambie et République de Guinée), qui alimentent un flux de migrations saisonnières vers le Sénégal oriental.

18 L'absence de relation entre les taux de ruralité et de migration nette dans les régions de Diourbel et de Tambacounda n'est en fait que l'illustration du déficit de corrélation entre ces deux variables sur le plan général. La valeur du coefficient de Bravais-Pearson n'est ici que de -0,325 et celle du coefficient de Spearman de -0,139, valeurs toutes deux non significatives : les régions les plus rurales ne sont en tout cas pas forcément celles dont on émigre le plus! Des facteurs économiques et/ou religieux peuvent interférer.

Néanmoins, une contingence apparaît entre taux de ruralité et taux de migration nette dans sept cas sur dix, suggérant malgré tout une relation de causalité entre les deux variables. Le calcul du coefficient de corrélation entre l'IDH et le taux de migration nette introduit une nuance supplémentaire. Le coefficient de corrélation par rang de Spearman entre ces deux variables est de $-0,210$, plus fort qu'entre les variables précédentes mais toujours non significatif et en tout cas beaucoup moins élevé qu'entre le niveau de l'IDH et le taux de ruralité.

Le faible niveau de l'IDH dans les régions sénégalaises en dehors de Dakar est tel que les écarts entre les régions ne sont pas d'une ampleur telle qu'ils généreraient automatiquement un flux migratoire interrégional consistant des régions les plus pauvres vers les régions les plus «nanties». A l'exception notable des migrations vers la région de Dakar, les courants migratoires interrégionaux revêtent souvent, là où ils existent, un caractère saisonnier lié aux activités agricoles. Leur incidence sur le taux de migration nette apparait ainsi dérisoire. Il s'y ajoute que toutes les régions sont peu ou prou des aires de diffluence humaine en direction de l'extérieur, notamment vers les pays développés. 


\section{La signification des différenciations régionales de la pauvreté}

21 Il résulte de ce qui précède que l'IDH, en tant que variable composite, doit être considérée comme l'indicateur fondamental de la pauvreté. Le taux de ruralité n'est qu'un indicateur de différenciation de deuxième ordre et les bilans migratoires ne sont que dans certains cas des révélateurs plutôt que des indicateurs des différenciations régionales de la pauvreté.

L'examen de la répartition spatiale de l'IDH, nuancée par celui des deux autres indicateurs, conduit donc à distinguer trois niveaux au Sénégal, en fonction de la pauvreté des régions (fig. 1).

La région de Dakar sera définie comme étant à pauvreté modérée. Celles de Ziguinchor, Diourbel, Thiès et Saint-Louis à pauvreté moyenne et les autres (Tambacounda, Kolda, Louga et Fatick) à pauvreté sévère. La carte oppose donc en gros l'ouest et le nord (vallée du Sénégal), modérément à moyennement pauvres, aux régions de l'est et du sud-est du pays, très pauvres. A l'ouest, la presqu'île du Cap-Vert, qui correspond à l'agglomération dakaroise et à sa région, vit une forme particulière de pauvreté liée à son caractère urbain. Les régions de Ziguinchor au sud et de Saint-Louis au nord doivent en partie leur position au caractère amphibie de leur territoire, dont le potentiel hydraulique leur vaut d'accueillir d'importants programmes d'aménagements hydroagricoles modernes destinés à réhabiliter les espaces de prospérité naguère façonnés le long des fleuves Sénégal et Casamance. Les autres régions (Thiès, Diourbel et Kaolack) coïncident avec le vieux bassin arachidier. L'espace de pauvreté sévère trace les contours des régions périphériques de Tambacounda et de Kolda à l'est et au sud-est et s'ouvre sous forme de biseaux sur les côtes nord et sud respectivement par la région semi-aride de Louga et par celle de Fatick, espace rural jadis polarisé par Kaolack et récemment érigé en région à la faveur d'une réforme administrative.

\section{Quelle lecture peut-on faire de cette configuration spatiale de la pauvreté?}

Le caractère rural de la pauvreté procède d'un certain nombre de facteurs dont on peut en retenir quatre, très imbriqués les uns les autres.

La décennie de sécheresse 1973-1984 et ses séquelles ont plongé une économie rurale encore largement dominée par l'agriculture pluviale dans un marasme généralisé. Sans doute cette sécheresse a-t-elle eu une durée et des impacts différents selon les régions écologiques; les parties septentrionales du pays en ont été les plus marquées. Néanmoins, elle a rendu vulnérable la quasi-totalité de l'espace national en l'exposant à la désertification. Même le sud humide ploie sous une surcharge démographique engendrée par l'afflux des populations des régions limitrophes, qui le fragilise de plus en plus.

Le dépérissement de l'économie de traite, qui reposait pour l'essentiel sur la monoculture de l'arachide, a laissé la plus grande partie des campagnes dans le désarroi. Le fait que la plupart des régions du bassin arachidier soient devenues des foyers d'émigration est symptomatique de l'absence et/ou de l'échec des alternatives de développement préconisées ou mises en oeuvre pour combler ce vide. 
27 La cure d'amaigrissement de l'Etat-providence imposée par les diverses facettes de la politique d'ajustement structurel de l'économie nationale inspirée par les institutions financières internationales depuis la fin des années quatre-vingt (Plan de Redressement Economique et Financier - PREF ; Plan d'Ajustement à Moyen et Long Termes - PAMLT ; Plan d'Ajustement Structurel - PAS) a porté le coup de grâce aux populations rurales en particulier, déjà fortement éprouvées par une succession de calamités naturelles (sécheresses récurrentes et invasions fréquentes de sauterelles) ainsi que par les séquelles de la logique d'exploitation du pacte colonial. Le désengagement drastique de l'Etat de larges secteurs de l'économie rurale et la prise en compte tardive des dimensions sociales de l'ajustement ont installé les campagnes sénégalaises dans un coma si profond qu'elles en émergent difficilement.

28 On peut aussi évoquer un facteur psychosociologique. Il serait lié aux capacités présumées plus grandes des masses urbaines à concevoir et à mettre en œuvre des stratégies de survie face à la crise. L'explosion au cours des dernières années du secteur informel, encore appelé économie populaire urbaine, est révélatrice de cette supposée plus grande faculté d'adaptation des citadins, ainsi que l'ont montré plusieurs recherches sur ce sujet (Antoine et al., 1995). Il y a dès lors urgence à réviser certains instruments macroéconomiques de calcul du niveau de vie dans les pays en développement, comme par exemple le PIB par habitant, qui reste une des bases fondamentales d'évaluation de l'IDH.

En conséquence, la signification des variations spatiales ne saurait se satisfaire de l'emprise exclusive des conditions naturelles puisque au demeurant la configuration régionale de la pauvreté n'épouse pas de manière absolue l'armature écologique du pays. Elle procède davantage de la reproduction à l'échelle du Sénégal des rapports de dépendance au plan international entre le centre et la périphérie, tissés par la logique de mise en place de l'économie-monde depuis de la découverte de l'Amérique. Le système monde dominé par l'Occident a pu fonctionner grâce à un réseau d'espacesrelais au sein de la périphérie. Saint-Louis et Dakar en furent des points stratégiques au sein de l'ex-Afrique occidentale française. L'accès à la souveraineté des différents pays de la sous-région a redimensionné ce sous-espace qu'il a recentré dans les limites du Sénégal indépendant. Celui-ci a hérité du système colonial des dysfonctionnements spatiaux qu'il n'a, jusqu'ici, jamais pu corriger.

Ces dysfonctionnements ont pour nom :

- la macrocéphalie de Dakar et plus généralement la primatie urbaine du Sénégal centralatlantique ;

- l'économie de traite ayant fixé les traits de l'infrastructure industrielle (constituée pour l'essentiel d'industries de substitution à l'importation), de transport (port, aéroport, lignes ferroviaires Dakar-Bamako, Dakar -Saint-Louis) et d'échanges ;

- le modèle jacobin d'administration territoriale, avec Dakar comme principal poste de commandement.

31 Cet héritage a fait du littoral le point de rupture de charge entre l'hinterland et l'extérieur, la métropole notamment. Le Sénégal atlantique est ainsi devenu tout naturellement l'espace de concentration urbaine et industrielle du pays. Cet espace s'est successivement dilaté vers la plaine alluviale du Sénégal à la faveur du développement des escales sur le fleuve, points de collecte de la gomme arabique, qui a alimenté principalement la navigation fluviale pendant plus d'un siècle, puis vers le centre lorsque la culture de l'arachide a supplanté le commerce de la gomme. Les 
régions urbaines du pays (le littoral, le bassin du fleuve, le bassin arachidier) coïncident au demeurant avec des espaces économiques de construction coloniale. Si le tissu urbain s'est distendu dans le nord et le centre du fait du déclin de l'économie de traite, il s'est en revanche densifié dans la presqu'île du Cap-Vert qui a conservé, entre autres, sa fonction de commandement, sur laquelle est venu se greffer le secteur informel, refuge de "nouveaux citadins » chassés des campagnes par une paupérisation accrue. Ces disparités spatiales de développement ont naturellement engendré des espaces de pauvreté différenciés par des déterminismes naturels historicisés.

\section{Quelles stratégies pour résorber par le haut les disparités régionales de la pauvreté ?}

En 1997, le gouvernement du Sénégal a adopté un Programme National de Lutte contre la Pauvreté (PNLP) dans la foulée du sommet de Copenhague consacré à ce problème. Le PNLP, à l'instar de beaucoup de ses homologues inspirés dans le monde par des bailleurs internationaux, conçoit cette lutte de façon sectorielle, en termes de ratios, de variables à améliorer (revenus, alphabétisation, couverture médicale, etc.), mettant souvent l'accent sur des catégories dites vulnérables (femmes, enfants, personnes âgées, etc.). Il n'appréhende pas explicitement le problème en termes de disparités régionales de développement à résorber. Aussi ne s'articule-t-il pas avec le Plan National d'Aménagement du Territoire (PNAT), dont l'une des vocations est de lutter contre ces inégalités.

Envisager le combat contre la pauvreté sous l'angle que voilà serait assurément un moyen de prévenir des frustrations qui peuvent naître ici et là et se cristalliser dans un réflexe identitaire pour se fondre dans un moule régional voire tribal. L'exacerbation de telles frustrations est, entre autres facteurs, à la base de l'irrédentisme casamançais dans le sud du pays. Il s'y ajoute qu'une stratégie spatiale appropriée pourrait contribuer à estomper voire juguler la pauvreté tout court.

Ces considérations générales suffisent-elles à fonder la pertinence d'imprimer à la lutte contre la pauvreté une dimension régionale dans le cadre d'une politique cohérente d'aménagement du territoire? Le Sénégal paraît s'être formellement engagé dans cette voie depuis l'adoption de la loi $72-25$ du 19 avril 1972, relative aux communautés rurales. Il vient de franchir un pas décisif dans ce processus de décentralisation, avec la promulgation, le 22 mars 1996, de la loi 96-06 portant code des collectivités locales que sont la région, la commune et la communauté rurale, désormais compétentes dans neuf domaines de la vie économique et sociale du pays jusque-là dévolus au pouvoir central. Cette loi dispose en son Titre II que les secteurs suivants relèvent désormais de la compétence des collectivités locales : les domaines; l'environnement et la gestion des ressources naturelles ; la santé, la population et l'action sociale ; la jeunesse, les sports et les loisirs; la culture ; l'éducation ; la planification ; l'aménagement du territoire ; l'urbanisme et l'habitat. Les collectivités locales, qui jouissent de l'autonomie financière, supportent les charges découlant de ce transfert. Leurs budgets sont alimentés par les ressources de la fiscalité locale pour les communes et les communautés rurales et par le Fonds de Dotation de la Décentralisation alloué par l'Etat à toutes les collectivités locales. Les critères de répartition de ce Fonds sont définis chaque année par décret. Les récriminations fréquentes des élus locaux dans la presse, se plaignant de l'insuffisance des moyens dont ils disposent, laissent croire que 
les budgets suffisent à peine à assurer le fonctionnement régulier des instances des collectivités locales pour laisser quelque recette à l'investissement dans l'un quelconque des secteurs de compétence ci-dessus. Au surplus les régions sont jetées dans cette politique de développement local avec des armes inégales, vu leur différence de niveau et de potentiel de développement originels. Ces inégalités de départ dans la capacité à réaliser les objectifs de développement de la réforme portent les germes de l'accroissement des disparités régionales de la pauvreté.

Une discrimination positive sous la forme d'un programme de développement spécial au profit des régions à pauvreté sévère pourrait contribuer à résorber progressivement les disparités régionales. Les ressources de ce programme proviendraient d'un fonds dénommé "Fonds spécial de développement local " alimenté, entre autres crédits substantiels alloués par divers bailleurs à la lutte contre la pauvreté, par une hausse de $1 \%$ du prélèvement actuel sur la TVA au profit du Fonds de Dotation de la Décentralisation. Le programme pourrait bénéficier de l'appui de la coopération décentralisée dont les actions et contributions seraient prioritairement orientées vers ces régions qu'il urge d'ériger en régions d'intervention prioritaire.

Dans ce contexte, la présente recherche, qui a permis de cerner les contours régionaux de la pauvreté au Sénégal, offre une base pour explorer et appliquer des stratégies de résorption positive des disparités régionales face à la misère.

\section{BIBLIOGRAPHIE}

ADAMS A. (1977), Le long voyage des gens du fleuve, Paris, Maspero.

ANTOINE Ph. \& al. (1995), Les Dakarois face à la crise, Dakar, IFAN.

BA M. (1995), Paysages et communautés au Sénégal oriental. Approche intégrée par cartographie et télédétection, Thèse de Doctorat, Université de Franche-Comté, Laboratoire Environnement Paysage - URA $908 \mathrm{du}$ CNRS, $407 \mathrm{p}$.

BANQUE MONDIALE (2001), Combattre la pauvreté, Rapport sur le Développement dans le monde, Paris, ESKA.

CENTRE D'ETUDES ET DE RECHERCHE SUR LA POPULATION ET LE DEVELOPPEMENT (CERPOD)

(1997), Enquête sur les migrations et l'urbanisation au Sénégal (EMUS) 1992-1993, Bamako.

CLARK S. \& al. (1991), « On indices for measurement of poverty 1981 », The Economic Journal, pp. 516-526.

DIRECTION DE LA PREVISION ET DE LA STATISTIQUE (DPS) (1991), Enquêtes sur les Priorités (EPS), Dakar.

DIRECTION DE LA PREVISION ET DE LA STATISTIQUE (DPS) $(1992,1996,1998)$, Enquêtes

démographiques et santé (EDS), Dakar.

DIRECTION DE LA PREVISION ET DE LA STATISTIQUE (DPS) (1995), Enquête sénégalaise auprès des ménages (ESAM), Dakar. 
LACHAUX J.-P. (1999), Concepts, mesures et analyse de la pauvreté en Afrique, Atelier régional sur l'utilisation des données sociales en politique de lutte contre la pauvreté, Cameroun 9-14 décembre 1998, Yaoundé.

PROGRAMME DES NATIONS UNIES POUR LE DEVELOPPEMENT (PNUD) (1997), Rapport mondial sur le développement humain, Paris, Economica.

PROGRAMME DES NATIONS UNIES POUR LE DEVELOPPEMENT (PNUD) (1998), Rapport sur le développement humain, Dakar.

PRADHAM M. \& RAVALLION M. (1998), Measuring Poverty Using Qualitative Perception of Welfare, Policy Research Working Paper, Document $n^{\circ}$ 2001, Development Research Group, Poverty and Human Resources, World Bank, Washington D.C.

RAVALLION M. \& LOKSHIN M. (1999), Subjective Economic Welfare, Policy Research Working Paper, Document $n^{\circ} 2106$, Development Research Group, Poverty and Human Resources, World Bank, Washington D.C.

ROBB C.M. (1992), Can the Poor influence Policy?, Participatory Poverty Assessments in the Developing World. Direction in Development, The World Bank, Washington D.C.

\section{NOTES}

1. Menée en 1991, l'ESP est une enquête par sondage. Elle a touché 9960 ménages, répartis sur toute l'étendue du territoire sénégalais, dont elle a étudié des caractéristiques socioéconomiques. Son objectif était d'identifier les groupes socio-économiques les plus vulnérables face au Plan d'Ajustement Structurel (PAS) de l'économie du Sénégal, imposé par le Fonds Monétaire International et la Banque Mondiale.

2. L'Enquête auprès des Ménages, publiée en 1995, est consécutive à "l'ajustement interne » adopté par les pays de la zone franc. Celui-ci, intervenu en janvier 1994, est marqué par un changement de parité entre le franc CFA et le franc français. L'ESAM a touché 3300 ménages dont elle a, comme l'enquête précédente, dégagé les principales caractéristiques socioéconomiques.

3. Les Enquêtes Démographiques et Santé (EDS I, II \& III) sont des enquêtes intercensitaires par sondage visant à suivre l'évolution des caractéristiques socio-démographiques de la population du Sénégal. A l'opposé des précédentes, elles renseignent plus sur les individus que sur les ménages. Les méthodes de collecte et de lecture des données sont également différentes. L'opposition ville - campagne n'y est pas systématisée comme grille d'analyse, ce qui complique la comparaison des résultats avec ceux des autres enquêtes.

4. Le concept d'IDH a été formulé en 1990 dans le premier Rapport sur le développement humain publié par le PNUD. Il a été conçu pour mettre en évidence que le développement économique, mesuré par les concepts classiques de la macroéconomie, comme le PIB/habitant, ne coïncide pas automatiquement avec le développement humain, qui doit aussi considérer des variables à caractère socio -culturel comme l'alphabétisation et l'espérance de vie. L'IDH intègre trois composantes : l'éducation, mesurée aux deux-tiers par le taux d'alphabétisation des adultes et au tiers par le taux de scolarisation toutes catégories confondues, la longévité, mesurée par l'espérance de vie à la naissance, et le niveau de vie par le PIB réel par habitant. L'IDH est constitué de la moyenne arithmétique de ces trois composantes. Il est mesuré sur une échelle graduée d'une valeur minimale de 0 et d'une valeur maximale de 1 pour chaque critère.

Avec un IDH moyen de 0,342 ( 0,772 pour la moyenne mondiale et 0,586 pour celle des pays en développement), le Sénégal occupe le 158ème rang sur 174 pays classés (Programme des Nations- 
Unies pour le Développement, 1998). Cette place fait du Sénégal, selon la typologie adoptée, un pays de «faible développement humain », en dépit des performances réalisées en termes de PIB, où le Sénégal occupe un meilleur rang - 132e sur 175 - . Il faudrait cependant nuancer la validité de l'IDH par les déficits de prise en compte du poids du secteur informel dans le calcul du PIB par habitant.

5. L'utilisation de ces deux derniers indicateurs a été suggérée par le Sommet Mondial sur l'Enfance (Rapport final, 1997).

6. Qu'il faut préférer au coefficient de Bravais-Pearson, dans la mesure où, d'une part, l'IDH n'est pas un indice dont la variation est linéaire et où, d'autre part, la répartition des régions en fonction du pourcentage de ruraux est très loin de répondre à une distribution normale, du fait de Dakar.

7. La région de Dakar coïncide avec l'agglomération du même nom et ne compte donc qu'un très faible pourcentage de ruraux, constitués de maraîchers, d'aviculteurs, de pêcheurs, etc., disséminés dans les faubourgs de la ville.

\section{RÉSUMÉS}

Un consensus n'a pas été trouvé à ce jour tant sur les contours du concept de pauvreté que sur les instruments qui permettraient d'en mesurer l'étendue. Il s'y ajoute que l'étude de la pauvreté souffre au Sénégal, peut-être plus qu'ailleurs, d'un déficit d'approche spatiale. Aussi, le phénomène est-il envisagé ici sous cet angle. La spatialisation esquissée débouche sur la proposition d'un dispositif de résorption des disparités régionales de la pauvreté pour plus de justice spatiale, une des finalités de la décentralisation, socle de la politique de développement local initiée au Sénégal depuis une trentaine année.

To date neither the outline of the concept of poverty nor the tools to assess the extent of poverty have been agreed on. In addition, in Senegal a spatial approach in the study of poverty is lacking maybe more than elsewhere. This is why we shall look into the phenomenon from that very angle, and propose a system to reduce the regional disparities of poverty aimed at more spatial justice, one of the goals of decentralisation which is the basis of the local development policy initiated in Senegal for about 30 years.

\section{INDEX}

Keywords : Senegal, poverty, human development indicators, migrations, regional disparities Mots-clés : Sénégal, pauvreté, indicateurs de développement humain, migrations, disparités régionales

\section{AUTEUR}

\section{AMADOU M. CAMARA}

Université Cheikh Anta Diop, B.P. 5005 Dakar-Fann, Sénégal, uvadakar@uva.org 